OF THE

\title{
KOREAN CHEMICAL SOCIETY
}

\section{Communications}

\section{$N, N^{\prime}$-Dibromo- $N, N^{\prime}$-1,2-ethanediylbis( $p$-toluenesulphonamide) as a Useful Reagent for Oxidation of 1,3,5-Trisubstituted Pyrazolines}

\author{
Ramin Ghorbani-Vaghei," Davood Azarifar, and Behrooz Maleki \\ Department of Chemistr?. Faculty of Science, Bu-Ali Sina University, IIamadan 65/74, Iran \\ Received December 17.2003
}

Key Words : Pyrazoline, Oxidation. BNBTS

The oxidation of 1,3.5-trisubstituted pyrazolines to pyrazoles is a very important reaction. Pyrazoles are widely used as analgesic, anti-inflamınatory, antipyretic, antiarrhythmic, tranquilizing, muscle relaxant, psychoanaleptic, anticonvulsant. monoamineoxidase inhibitor, antidiabetic and antibacterial activities. ${ }^{1}$ 1,3,5-'] risubstituted pyrazolines can be easily prepared from phenylhydrazine and chalcone derivatives. ${ }^{2}$ Oxidative aromatization of pyrazolines, with oxidizing reagents provides an efficient method for the preparation of pyrazole derivatives. Several reagents such as $\mathrm{Zr}\left(\mathrm{NO}_{3}\right)_{1},{ }^{3} \mathrm{Pd} / \mathrm{C}$, $\mathrm{Co}(\mathrm{II})$ and oxygen, ${ }^{5}$ iodobenzene diacetate, ${ }^{6}$ lead tetraacetate, ${ }^{7} \mathrm{MnO}_{2}{ }^{8}$ potassium permanganate" and NBS, ${ }^{10}$ have been reported for the preparation of pyrazoles. It is note worthy to say that most of the procedures have been developed for this purpose suffer from some disadvantages including need to use excess reagent, long reaction times, high temperatures, formation of side products and difficulty to remove the reagent from the sensitive pyrazoles.

Herein, we report on a simple and efficient procedure for oxidative aromatization of $1.3,5$-substituted pyrazolines to pyrazoles using $N, N^{\prime}$-dibromo- $N, N^{\prime}$-1,2-ethanediylbis ( $p$ toluenesulphonamide) [BNBTS| (Fig. l).

The advantages of BNBTS are as follows:

1. It can be prepared easily.

2. It is stable in atmospheric conditions for two months.

3. After completing the reaction and evaporation of solvent, the sulphonamide can be recovered and reused many times without decreasing the yield.

"Coresponding Author. Fax: (-98) 811 8272404; c-mail: ghorbaniabasu.ac.ir<smiles>C#Cc1ccc(S(=O)(=O)N(C)CCCCC)cc1</smiles>

1 BNBTS

ligure I

The reaction of 1,3,5-trisubstituted pyrazolines with BNBTS in carbon tetrachloride at room temperature afforded pyrazoles without side products (Scheme 1).

The results of the conversion of various 1,3,5-trisubstituted pyrazolines to their corresponding pyrazoles are presented in lable 1 .

Since BNBTS contains two bromine atoms which are attached to nitrogen atoms it is very probable that this reagent releases $\mathrm{Br}^{+}$in situ which can conduct as an electrophilic species. ${ }^{11}$ [herefore the following mechanism<smiles>[R]C1=NN(c2ccccc2)C([R])C1</smiles>

3<smiles>[R]c1cc([R])n(-c2ccccc2)n1</smiles>
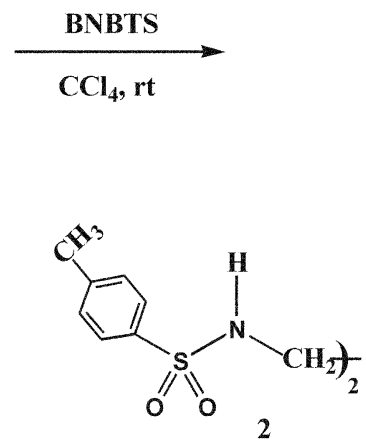

Scheme 
<smiles>[R]C1=NN(c2ccccc2)C([R])C1</smiles><smiles>C#Cc1ccc(S(=O)(=O)N(C)Br)cc1</smiles><smiles>[R]C1=NN(c2ccccc2)C([R])CC1[R]</smiles><smiles>[R]c1cc([R])n(-c2ccccc2)n1</smiles><smiles>[R]C1=NN(c2ccccc2)C([R])C1(CC)CC</smiles><smiles>[R]C1=NN(c2ccccc2)C([R])C1</smiles><smiles>C#Cc1ccc(S(=O)(=O)NC)cc1</smiles>

Scheme 2

Table 1. Aromatization of 1.3.5-trisubstituted pyrazolines with BNBTS

\begin{tabular}{|c|c|c|c|c|c|c|}
\hline Subsirate & Product" & $\mathrm{R}^{1}$ & $\mathrm{R}^{2}$ & Reagentil'roduct & lïme (min) & Yield $(\%)$ \\
\hline $3 a$ & 4a & 2-Naphthyl & $2-\mathrm{Cl}_{3} \mathrm{C}_{6} \mathrm{H}_{4}$ & 1.25 & 20 & 92 \\
\hline $3 \mathbf{b}$ & $4 b$ & 2-Naphthyl & $\mathrm{Ph}$ & 1.25 & 25 & 86 \\
\hline $3 c$ & $4 \mathrm{c}$ & Ph & $\mathrm{Ph}$ & 1.25 & 15 & 82 \\
\hline $3 d$ & $4 d$ & 4-CH $\mathrm{C}_{4} \mathrm{I}_{3}$ & $3-\mathrm{Cl}_{3} \mathrm{C}_{6} \mathrm{H}_{4}$ & 2.5 & 20 & 90 \\
\hline $3 e$ & $4 e$ & 3-CII $\mathrm{C}_{4} \mathrm{II}_{3}$ & $2-\mathrm{ClC}_{6} \mathrm{H}_{4}$ & 2.5 & 20 & 89 \\
\hline $3 f$ & $4 f$ & ${ }_{4}-\mathrm{OCH}_{3} \mathrm{C}_{6} \mathrm{H}_{4}$ & $3-\mathrm{Cl}_{3} \mathrm{C}_{6} \mathrm{H}_{4}$ & 2 & 15 & 86 \\
\hline $3 \mathbf{g}$ & $4 \mathrm{~g}$ & $4-\mathrm{OCH}_{3} \mathrm{C}_{6} \mathrm{H}_{4}$ & $2-\mathrm{ClI}_{3} \mathrm{C}_{5} \mathrm{H}_{4}$ & 1.75 & 20 & 82 \\
\hline $3 \mathbf{h}$ & $4 \mathrm{~h}$ & $4-\left(\mathrm{Cll}_{3} \mathrm{C}_{6} \mathrm{H}_{4}\right.$ & $\mathrm{Ph}$ & 1.75 & 15 & 80 \\
\hline $3 \mathbf{i}$ & $4 i$ & $4-() \mathrm{Cll}_{3} \mathrm{C}_{6} \mathrm{H}_{6}$ & $4-\mathrm{ClC}_{6} \mathrm{H}_{6}$ & 2,25 & 20 & 78 \\
\hline $3 \mathbf{j}$ & $4 j$ & 3-CII $\mathrm{C}_{4} \mathrm{IH}_{4}$ & $4-\mathrm{ClC}_{6} \mathrm{H}_{6}$ & 2.25 & 20 & 86 \\
\hline $3 k$ & $4 k$ & 2-Naphthyl & $3-\mathrm{Cl}_{3} \mathrm{C}_{6} \mathrm{H}_{4}$ & 2 & 15 & 80 \\
\hline $3 \mathbf{I}$ & 41 & 2-Naphthyl & $4-\mathrm{ClC}_{6} \mathrm{H}_{4}$ & 2.5 & 25 & 70 \\
\hline $3 m$ & $4 m$ & 2-Naphthyl & $2-\mathrm{ClC}_{6} \mathrm{H}_{-6}$ & 2,25 & 15 & 68 \\
\hline 3n & $4 n$ & $3-\mathrm{C}_{3} \mathrm{C}_{4} \mathrm{H}_{3}$ & $4-\lambda\left(C\left(I_{3}\right)_{2} C_{0} I_{1}\right.$ & 2.25 & 20 & 70 \\
\hline 30 & 40 & 2-( $\mathrm{CII}_{3} \mathrm{C}_{4} \mathrm{II}_{3}$ & $4-\Lambda\left(C \cdot H_{3}\right)_{2} C_{0} I_{1}$ & 2,25 & 20 & 68 \\
\hline $3 p$ & $4 p$ & $4-() \mathrm{Cl}_{3} \mathrm{C}_{6} \mathrm{H}_{6}$ & $2-\mathrm{ClC}_{6} \mathrm{H}_{-6}$ & 1.75 & 20 & 85 \\
\hline
\end{tabular}

"I'toducts were characterized by their physical properties. comparison with authentic samples and by spectroscopic methods.

can be suggested for the conversion 1,3,5-trisubstituted pyrazolines to pyrazoles (Scheme 2).

IR and NMR spectra were recorded using a Shimadzu 435-U-04 spectrophotometer (KBr pellets) and a $90 \mathrm{MHz}$ Jeol FT NMR spectrometer, respectively.

General procedtre for oxidation of 1,3,5-strbstituted pyrazolines with BNBTS: To a solution of 1,3,5-substituted pyrazoline 3 (2 mmol) in $\mathrm{CCl}_{1}(10 \mathrm{~mL})$ was added BNBTS (molar ratio given in Table 1). The reaction mixture was stirred at room temperature for the time given in Table 1. After complete conversion as indicated by $\mathrm{TLC}, \mathrm{K}_{2} \mathrm{CO}_{3}(0.5$ g) was added and stirred for $0.5 \mathrm{~h}$, the insoluble sulphonamide 2 was removed by filtration and washed with cold $\mathrm{CCl}_{4}(5 \mathrm{~mL})$. Removal of the solvent under reduced pressure gave the crude product. The pure product 4 was obtained by recrystallization with methanol $/ \mathrm{H}_{2} \mathrm{O}(\mathrm{IO}: 1)$.

\section{References}

1. (a) Takabata E.: Kodama. R.: Tanaka Y.: Dohmori. R.:
Tachizawa. H.: Vaito. T. Chem. Phum. Bull. 1979. /6. 1900. (b) Parmar S. S.: Pandey B. R.: Dwivedi. C. I Iarbison, R. D. J Phom Sci. 1974. 63. 1152. (c) Soni. N.: Pande. K.: Kalsi. R.: Cuptal T. K.: Parmer. S. S.: Barthwal. I. P. Res. Commun. Chem. Pathol. Phatrm. 1987. 56. 129.

2. (a) Azarifar. D.: Shacbanzadch. M. Molecules 2002. 7. 885. (b) Azarifar. D.: Ghasemnejad. H. Holecules 2003. \$. 642.

3. Sabithar G.: Reddy: G. S. K. K.: Reddy: C. S.: Fatima N.: Yadav. J. S. Symthesis 2003.1267.

4. Nakanichi. N.: Kawashita. Y. Hayatshi. M. Org. Letf. 2002. t. 3955.

5. Shah. J. N.: Shah. C. K. J. Org. Chem. 1978. f3. 1266

6. Singh. S. P.: Kumar. D.: Prakash. O.: Kapoor. R. P. Synth. Conmmin. 1997, 27, 2683.

7. Gladitome. W. A. F. Norman. R. (). C. . Chen. Soc. Chem. (ommin. 1966. 1536.

8. Bhatnagar. I.: George. M. V. Tetruthetron 1968. 2f. 1293.

9. Smith. L. I.: Howard. K. L. J. Am. Chem Soc 1943. 65. 159.

10. Ritd. W.: lantzech. R. Chem. Bes: 1969. 102, 378 .

11. (a) Khavadi. A.: Shirdarrel. A. Sinth. Conmm 1999. 29. 4079. (b) Khazati. A.: Vaghei. R. (i.: Iajpakhsh. M. Tetrahedron lett. 2001. +2. 5099. (c) Ghorbani-Vaghei. R.: Khazaei. A. Tetrohedron Lett. 2003. 44. 7525 . 